\title{
Effect of Germination on Physico-chemical Properties of Rice Varieties
}

\author{
Soubhagyalaxmi P. Hiremath* and B. Kasturiba \\ Department of Food Science and Nutrition, College of Community Science, University of \\ Agricultural Sciences, Dharwad, Karnataka, India \\ *Corresponding author
}

\section{A B S T R A C T}

\begin{tabular}{|l|}
\hline Ke y w or d s \\
Rice, Physical, \\
Chemical, \\
Germination
\end{tabular}

\section{Introduction}

Rice (Oryza sativa L.), a member of the grass family, is one of the crop on which the human species largely subsists. Rice is now one of the most important crops at the global level, as it is used as a staple food in most countries of the world. Brown Rice is the unpolished whole grain rice that is produced by removing only the hull or husk using a motar and pestle or rubber rolls, having a mild nutty flavour, chewier than white rice. Colour of rice kernel may be brown, reddish, purplish or blackish in color. Brown rice is more nutritious than white rice but its intake is limited because of its chewy texture and reduced digestibility. This problem can be overcome by subjecting rice to germination, thus, producing germinated rice. Germination is a process involved in incorporating those events that commence with the uptake of water by a quiescent dry seed and terminate with the elongation of the embryonic axis. It signals the birth of a new life. At the time of germinating, huge amounts of nutrients are prepared for the growth of sprout. The birth of the sprout activated all the dormant enzymes in the rice in order to supply the sprout with the best nutrition. Rice quality differs according to the variety and processing method used. Quality desired in rice vary from one geographical region to another and consumer demand certain varieties and favors 
specific quality traits of milled rice for home cooking (Danbaba et al., 2011). Physical properties of the grain are those which are recognizable in the marketplace. All physical qualities can be affected by the growth conditions of the plant, in particular high temperatures during grain filling, field fertilization and harvest moisture. The gelatinization temperature (GT), gel consistency (GC) and amylose content (AC) are major rice traits, which are directly related to cooking and eating quality. It is known that different germination conditions would affect the properties of ricedifferently but positive results in terms of functional properties were obtained (Musa et al., 2011). Many functional properties of rice are believed to be closely related to physicochemical properties. Hence the present study was undertaken with objective to determine the effect of germination on physico-chemical properties of rice varieties.

\section{Material and Methods}

Ten rice varieties viz., Abhilash, Ambemohar 2, Dodiga, Ginasali, Hasudi, Intan, Jyoti, Karikalavi, Karihakkalasali and Navaliwere procured from Agricultural Research Station (ARS), Mugad. Rice was prepared by removing husk of the paddy using a McGill dehuller.

Milling of paddy samples: $125 \mathrm{~g}$ of paddy sample was used for milling determinations. Whole grains (head rice) were separated from total milled rice and resulting head rice was weighed. Broken grains were separated from total milled rice and resulting broken rice was weighed (Singh et al., 2000).

Preparation of germinated rice: Preliminary trials were carried out to determine the grain to water ratio for soaking of rice grains. Trials were carried out to optimize the time and temperature to attain $0.5-1 \mathrm{~cm}$ sprouts during germination. Whole rice grains were washed and soaked in distilled water (grain-to-water ratio $1: 3, W / V$ ) at $30^{\circ} \mathrm{C}$ for $12 \mathrm{~h}$ and kept for germination in incubator at $30^{\circ} \mathrm{C}$ for $24 \mathrm{~h}$. Germinated rice with $0.5-1.0 \mathrm{~cm}$ sprouts was dried in hot air oven at $50^{\circ} \mathrm{C}$.

Per cent germination of rice grains: 100 seeds were placed in a petriplate on a filter paper dampened with water and the lid was closed, kept in incubator and allowed to germinate for $24 \mathrm{hrs}$. Further germinated seeds were counted and expressed in percentage (Aykroyd and Doughty, 1964).

Length and Breadth of randomly picked 10 full grains per replication was measured using Verniercalliper. The L/B ratio was calculated by dividing the cumulative length of 10 kernels by the cumulative breadth of 10 kernels. Based on the L/B ratio, grains were classified into long slender (LS), short slender (SS), medium slender (MS), long bold (LB) and short bold (SB).Representative samples of each variety were drawn randomly and thousand kernel weight was recorded in grams/1000 kernels by counting grains and weighed on an electronic balance. The volume of the thousand kernel was determined by water displacement method. Bulk density was determined using mass/volume relation. Specific gravity of rice grains was determined using specific gravity bottle and toluene solution (William et al., 1983).

\section{Amylose content}

Hundred $\mathrm{mg}$ of rice flour was placed in 100 $\mathrm{ml}$ volumetric flasks and added $1 \mathrm{ml}$ of $95 \%$ $\mathrm{v} / \mathrm{v}$ ethanol. Then $9 \mathrm{ml}$ of $40 \mathrm{~g} \mathrm{NaOH}$ dissolved in one liter was added and heated in a boiling water bath for $10 \mathrm{~min}$. Samples were diluted to $100 \mathrm{ml}$ with distilled water. Later, 5 $\mathrm{ml}$ of sample suspension was added to $50 \mathrm{ml}$ of distilled water in a $100 \mathrm{ml}$ of flask and $1 \mathrm{ml}$ of acetic acid (57.75 ml in one liter water) was 
added to acidify the sample along with $1.5 \mathrm{ml}$ of iodine solution ( 0.2 percent $\mathrm{w} / \mathrm{v}$ iodine in $2 \%$ potassium iodide). Distilled water was added to make the volume of $100 \mathrm{ml}$ and the suspension was mixed well and kept for 20 min. As a control, $\mathrm{NaOH}$ solution was used for the calibration of spectrophotometer and samples were measured at $620 \mathrm{~nm}$. Samples with known values of high, medium and low AC were used to draw the standard AC curve. The AC of different varieties was calculated in comparison with standard graph (Bhonsle and Sellapan, 2010).

\section{Alkali spread value}

Six milled rice were taken in petri plates and $10 \mathrm{ml}$ of potassium hydroxide (19.54 $\mathrm{g}$ of potassium hydroxide dissolve in one liter) was added to the sample. Samples were kept undisturbed for 23 hours in an incubator at 27$30^{\circ} \mathrm{C}$. The spreading of each grain was rated visually on a 7-point numerical scale.

\section{Gel consistency}

Milled rice was ground to a fine powder using mortar \& pestle and sieved with $1 \mathrm{~mm}$ sieve. $100 \mathrm{mg}$ of rice flour was taken in long test tube $(2 \times 19.5 \mathrm{~cm})$ and added $0.2 \mathrm{ml}$ of ethanol containing $0.25 \%$ thymol blue and $2.0 \mathrm{ml}$ of $2.8 \mathrm{~g}$ of $\mathrm{KOH}$ in $250 \mathrm{ml}$ distilled water was added and mixed well using vortex mixture, kept in boiling water bath for $8 \mathrm{~min}$, cooled for $5 \mathrm{~min}$, mixed and kept in ice bath for 20 min. Later tubes were removed, laid horizontally for one hour and measurements were made using graph paper (Bhonsle and Sellapan, 2010).

\section{Statistical analysis}

All experiments were carried out using three replicates of each sample for analyses. The results were subjected to analysis of variance (ANOVA). Means were compared by
Duncan's multiple range test (DMRT). Difference between raw and germinated rice was evaluated. A level of significance of 0.05 and 0.01 was used.

\section{Results and Discussion}

Milling characteristics of rice i.e., head rice, broken and husk yield are presented in Figure 1. Head rice yield, brokens and husk per cent among rice varieties differed significantly $(\mathrm{P} \leq 0.01)$. Highest head rice yield of 59.33 per cent was obtained from Ambemohar 2 and lowest head rice yield of 11.33 per cent was obtained in Dodiga. Broken rice yield ranged from 16.00 to 60.00 per cent. Husk yield in rice varieties ranged from 20.33 to 32.33 per cent. Head rice value depends on the grain type, chalkiness, cultivation practices and drying conditions (Bhonsle and Sellappan, 2010). The relatively high percentage of broken fractions could be attributable to low moisture content (Gayin et al., in 2009).

Per cent germination of rice varieties is presented in Figure 2. The mean per cent germination of rice varieties ranged from 77.33 to 99.67 per cent. There was significant difference found among the rice varieties for per cent germination $(\mathrm{P} \leq 0.01)$. Highest per cent germination of 99.67 per cent was found in Ambemohar2 followed by Hasudi (96.33 per cent) and lowest per cent germination of rice grains was determined in Dodiga i.e., 77.33 per cent. Similar results were found in a study conducted by Moongngram and Khomphiphatkul (2011). The germination rate reached the maximum (88.33-99.33 per cent) after germination for longer than 2 days. The length of shoot and root of germinated seeds were increased $(\mathrm{p} \leq 0.05)$ with germination times.

Physical properties of raw and germinated rice varieties such as length, breadth, L/B ratio, thousand kernel weight, thousand kernel 
volume, bulk density and specific gravity are presented in Table $1 \mathrm{a}$ and $\mathrm{b}$. The mean length of rice ranged from 3.77 to $6.20 \mathrm{~mm}$ and the mean length of germinated rice ranged from 4.11 to $6.21 \mathrm{~mm}$. Length of the rice grains significantly differed among the varieties $(\mathrm{P} \leq 0.05)$ but there was no significant difference found in the breadth and L/B ratio of rice varieties. Size expansion of germinated rice was noticeable with longer germination time, leading to decreased $\mathrm{L} / \mathrm{B}$ ratio (Jiamyangyuen and Ooraikul, 2008). Based on $\mathrm{L} / \mathrm{B}$ ratio, raw rice variety Intan (3.03) was slender; Abhilash (2.27), Ambemohar2 (2.29), Ginasali (2.26), Hasudi (2.06), Jyoti (2.95), Navali (2.28) were medium grains; Dodiga (1.93), Karikalavi (1.97), Karihakkalasali (1.90) were bold grains. The mean thousand kernel weight of germinated rice varieties of
$21.91 \mathrm{~g}$ was found to be higher than the mean thousand kernel weight of raw rice varieties $(21.78 \mathrm{~g})$. Germinated rice variety, Karihakkasali had highest thousand kernel volume i.e., $33.00 \mathrm{ml}$ whereas germinated rice variety, Ambemohr-2 had lowest thousand kernel volume of $13.66 \mathrm{ml}$. Significant difference was found in thousand kernel weight and volume of rice grains among the varieties and between raw and germinated $(\mathrm{P} \leq 0.01)$. Rice soaked for $12 \mathrm{~h}$ showed higher thousand kernel weight (21.64 g), compared to ungerminated sample (19.5 g) (Jiamyangyuen and Ooraikul, 2008). The mean bulk density of raw rice varieties i.e., $0.81 \mathrm{~g} / \mathrm{ml}$ was found to be higher than the mean bulk density of germinated rice varieties i.e., $0.79 \mathrm{~g} / \mathrm{ml}$. Rice varieties varied significantly with respect to bulk density of rice grains $(\mathrm{P} \leq 0.01)$.

Table.1a Physical properties of raw and germinated rice varieties

\begin{tabular}{|c|c|c|c|c|c|c|}
\hline \multirow{2}{*}{$\begin{array}{l}\text { Property } \\
\text { Variety }\end{array}$} & \multicolumn{2}{|c|}{ Length(mm) } & \multicolumn{2}{|c|}{ Breadth $(\mathrm{mm})$} & \multicolumn{2}{|c|}{ L/B ratio } \\
\hline & Raw & Germinated & Raw & Germinated & Raw & Germinated \\
\hline Abhilash & $6.07 \pm 0.18^{\mathrm{e}}$ & $6.08 \pm 0.12^{\mathrm{fg}}$ & $2.66 \pm 0.07^{\mathrm{d}}$ & $2.71 \pm 0.10^{\mathrm{cd}}$ & $2.27 \pm 0.09^{b}$ & $2.24 \pm 0.06^{\mathrm{cd}}$ \\
\hline Ambemohar 2 & $4.79 \pm 0.21^{\mathrm{b}}$ & $4.92 \pm 0.16^{b}$ & $2.08 \pm 0.06^{b}$ & $2.12 \pm 0.01^{\mathrm{b}}$ & $2.29 \pm 0.10^{b}$ & $2.31 \pm 0.07^{\mathrm{d}}$ \\
\hline Dodiga & $5.53 \pm 0.04^{\mathrm{c}}$ & $5.68 \pm 0.12^{\mathrm{c}}$ & $2.80 \pm 0.10^{\mathrm{e}}$ & $2.84 \pm 0.16^{\mathrm{de}}$ & $1.93 \pm 0.03^{\mathrm{a}}$ & $2.00 \pm 0.12^{\mathrm{ab}}$ \\
\hline Ginasali & $6.20 \pm 0.07^{\mathrm{e}}$ & $6.21 \pm 0.07^{\mathrm{g}}$ & $2.73 \pm 0.04^{\mathrm{de}}$ & $2.77 \pm 0.07^{\mathrm{de}}$ & $2.26 \pm 0.04^{b}$ & $2.24 \pm 0.06^{\mathrm{cd}}$ \\
\hline Hasudi & $5.87 \pm 0.23^{\mathrm{cd}}$ & $6.07 \pm 0.19^{\mathrm{de}}$ & $2.84 \pm 0.05^{\mathrm{e}}$ & $2.88 \pm 0.09^{\mathrm{e}}$ & $2.06 \pm 0.10^{\mathrm{a}}$ & $2.10 \pm 0.07^{\mathrm{bc}}$ \\
\hline Intan & $6.15 \pm 0.08^{e}$ & $6.15 \pm 0.05^{\mathrm{fg}}$ & $2.00 \pm 0.06^{b}$ & $2.04 \pm 0.05^{\mathrm{b}}$ & $3.03 \pm 0.05^{\mathrm{c}}$ & $3.01 \pm 0.08^{\mathrm{e}}$ \\
\hline Jyoti & $3.77 \pm 0.50^{\mathrm{a}}$ & $4.11 \pm 0.36^{\mathrm{a}}$ & $1.27 \pm 0.04^{\mathrm{a}}$ & $1.27 \pm 0.01^{\mathrm{a}}$ & $2.95 \pm 0.29^{\mathrm{c}}$ & $3.23 \pm 0.04^{\mathrm{t}}$ \\
\hline Karikalavi & $6.01 \pm 0.07^{\text {de }}$ & $6.04 \pm 0.06^{\mathrm{de}}$ & $3.03 \pm 0.12^{\mathrm{f}}$ & $3.08 \pm 0.07^{\mathrm{g}}$ & $1.97 \pm 0.07^{\mathrm{a}}$ & $1.96 \pm 0.06^{\mathrm{a}}$ \\
\hline Karihakkalasali & $5.84 \pm 0.06^{\mathrm{cd}}$ & $5.93 \pm 0.07^{\mathrm{d}}$ & $3.05 \pm 0.05^{\mathrm{f}}$ & $3.08 \pm 0.05^{\mathrm{f}}$ & $1.90 \pm 0.05^{\mathrm{a}}$ & $1.92 \pm 0.03^{\mathrm{a}}$ \\
\hline Navali & $5.68 \pm 0.06^{\text {cd }}$ & $5.70 \pm 0.08^{c}$ & $2.49 \pm 0.01^{\mathrm{c}}$ & $2.57 \pm 0.13^{\mathrm{c}}$ & $2.28 \pm 0.01^{b}$ & $2.21 \pm 0.11^{\mathrm{cd}}$ \\
\hline Mean & $5.59 \pm 0.75$ & $5.69 \pm 0.70$ & $2.50 \pm 0.54$ & $2.53 \pm 0.55$ & $2.30 \pm 0.39$ & $2.23 \pm 0.43$ \\
\hline
\end{tabular}

\begin{tabular}{|c|c|c|c|c|c|c|c|c|c|}
\hline & SEM & CD & F value & SEM & CD & F value & SEM & CD & F value \\
\hline Variety & 0.063 & 0.180 & $*$ & 0.032 & 0.093 & $*$ & 0.039 & 0.113 & $*$ \\
\hline R x G & 0.028 & 0.080 & $*$ & 0.014 & 0.041 & NS & 0.017 & 0.051 & NS \\
\hline Interaction & 0.089 & 0.255 & $*$ & 0.046 & 0.132 & NS & 0.056 & 0.161 & NS \\
\hline
\end{tabular}

Note: Values are mean of three replications, SEm \pm - Standard error of mean, CD - critical difference, NS-Non significant, *Significant @ 5\%.Values with same letters (a, b , c, d, e, f, g) in the same column are not significantly different $(\mathrm{P} \leq 0.05)$.

R: Raw; G: Germinated 
Table.1b Physical properties of raw and germinated rice varieties

\begin{tabular}{|c|c|c|c|c|c|c|c|c|}
\hline \multirow{2}{*}{$\begin{array}{l}\text { Property } \\
\text { Variety }\end{array}$} & \multicolumn{2}{|c|}{1000 kernel weight $(\mathrm{g})$} & \multicolumn{2}{|c|}{1000 kernel volume (ml) } & \multicolumn{2}{|c|}{ Bulk density $(\mathrm{g} / \mathrm{ml})$} & \multicolumn{2}{|c|}{ Specific gravity } \\
\hline & Raw & Germinated & Raw & Germinated & Raw & Germinated & Raw & Germinated \\
\hline Abhilash & $23.95 \pm 0.18^{f}$ & $24.09 \pm 0.07^{f}$ & $30.00 \pm 0.00^{\mathrm{def}}$ & $30.33 \pm 0.57^{\mathrm{d}}$ & $0.80 \pm 0.01^{\mathrm{bc}}$ & $0.79 \pm 0.01^{\mathrm{c}}$ & $1.45 \pm 0.01^{\mathrm{e}}$ & $1.13 \pm 0.01^{\mathrm{e}}$ \\
\hline Ambemohar 2 & $10.16 \pm 0.00^{\mathrm{a}}$ & $10.19 \pm 0.02^{\mathrm{a}}$ & $13.00 \pm 0.00^{\mathrm{a}}$ & $13.66 \pm 0.57^{\mathrm{a}}$ & $0.78 \pm 0.00^{b}$ & $0.74 \pm 0.03^{b}$ & $1.27 \pm 0.01^{\mathrm{b}}$ & $0.99 \pm 0.01^{b}$ \\
\hline Dodiga & $26.18 \pm 0.38^{h}$ & $26.27 \pm 0.34^{\mathrm{gh}}$ & $30.33 \pm 0.57^{\mathrm{ef}}$ & $30.66 \pm 0.57^{\mathrm{de}}$ & $0.86 \pm 0.02^{\mathrm{e}}$ & $0.85 \pm 0.01^{\mathrm{d}}$ & $1.37 \pm 0.02^{\mathrm{c}}$ & $1.08 \pm 0.01^{\mathrm{c}}$ \\
\hline Ginasali & $23.44 \pm 0.00^{\mathrm{e}}$ & $23.46 \pm 0.01^{\mathrm{e}}$ & $30.00 \pm 0.00 \mathrm{~d}^{\mathrm{ef}}$ & $30.00 \pm 0.00^{\mathrm{d}}$ & $0.78 \pm 0.00^{b}$ & $0.78 \pm 0.00^{c}$ & $1.56 \pm 0.00^{\mathrm{g}}$ & $1.22 \pm 0.00^{\mathrm{g}}$ \\
\hline Hasudi & $22.26 \pm 0.02^{\mathrm{d}}$ & $22.30 \pm 001^{\mathrm{d}}$ & $26.66 \pm 0.57^{c}$ & $27.66 \pm 0.57^{\mathrm{c}}$ & $0.83 \pm 0.02^{\mathrm{d}}$ & $0.81 \pm 0.02^{\mathrm{c}}$ & $1.93 \pm 0.01^{\mathrm{i}}$ & $1.51 \pm 0.00^{\mathrm{i}}$ \\
\hline Intan & $17.46 \pm 0.07^{b}$ & $17.85 \pm 0.19^{b}$ & $18.00 \pm 0.00^{\mathrm{b}}$ & $18.33 \pm 0.57^{b}$ & $0.97 \pm 0.00^{f}$ & $0.97 \pm 0.02^{\mathrm{e}}$ & $1.38 \pm 0.01^{\mathrm{c}}$ & $1.08 \pm 0.00^{c}$ \\
\hline Jyoti & $21.01 \pm 0.08^{c}$ & $21.14 \pm 0.07^{\mathrm{c}}$ & $29.66 \pm 0.57^{\mathrm{de}}$ & $30.33 \pm 0.57^{\mathrm{d}}$ & $0.71 \pm 0.01^{\mathrm{a}}$ & $0.70 \pm 0.01^{\mathrm{a}}$ & $1.51 \pm 0.00^{f}$ & $1.18 \pm 0.00^{f}$ \\
\hline Karikalavi & $25.87 \pm 0.01^{\mathrm{g}}$ & $26.11 \pm 0.01^{\mathrm{g}}$ & $30.66 \pm 0.57^{f}$ & $31.33 \pm 0.57^{\mathrm{e}}$ & $0.84 \pm 0.02^{\text {de }}$ & $0.83 \pm 0.01^{\mathrm{d}}$ & $1.60 \pm 0.01^{\mathrm{h}}$ & $1.25 \pm 0.01^{\mathrm{h}}$ \\
\hline Karihakkalasali & $26.42 \pm 0.01^{\mathrm{h}}$ & $26.51 \pm 0.01^{\mathrm{h}}$ & $32.66 \pm 0.57^{\mathrm{g}}$ & $33.00 \pm 0.00^{f}$ & $0.81 \pm 0.01^{\mathrm{c}}$ & $0.80 \pm 0.00^{c}$ & $1.15 \pm 0.01^{\mathrm{a}}$ & $0.90 \pm 0.01^{\mathrm{a}}$ \\
\hline Navali & $21.04 \pm 0.23^{c}$ & $21.16 \pm 0.21^{\mathrm{c}}$ & $29.33 \pm 0.57^{\mathrm{d}}$ & $30.66 \pm 0.57^{\mathrm{de}}$ & $0.72 \pm 0.02^{\mathrm{a}}$ & $0.69 \pm 0.01^{\mathrm{a}}$ & $1.41 \pm 0.01^{\mathrm{d}}$ & $1.10 \pm 0.01^{\mathrm{d}}$ \\
\hline Mean & $21.78 \pm 4.77$ & $21.91 \pm 4.77$ & $27.03 \pm 6.15$ & $27.60 \pm 6.14$ & $0.81 \pm 0.07$ & $0.79 \pm 0.08$ & $1.46 \pm 0.20$ & $1.15 \pm 0.15$ \\
\hline
\end{tabular}

\begin{tabular}{|c|c|c|c|c|c|c|c|c|c|c|c|c|c|}
\hline & SEM & CD & F value & SEM & CD & F value & SEM & CD & F value & SEM & CD & F value \\
\hline Variety & 0.062 & 0.177 & $* *$ & 0.197 & 0.563 & $* *$ & 0.006 & 0.017 & $* *$ & 0.003 & 0.008 & $* *$ & $*$ \\
\hline R x G & 0.027 & 0.079 & $* *$ & 0.088 & 0.252 & $* *$ & 0.002 & 0.007 & $* *$ & 0.001 & 0.003 & $* *$ \\
\hline $\begin{array}{c}\text { Interacti } \\
\text { on }\end{array}$ & 0.087 & 0.250 & $* *$ & 0.278 & 0.797 & $* *$ & 0.008 & 0.024 & $* *$ & 0.004 & 0.012 & $* *$ \\
\end{tabular}

Note: Values are mean of three replications, SEm \pm - Standard error of mean, CD - critical difference, **Significant @ $1 \%$. Values with same letters (a, b, c, d, e, f, g, h, i) in the same column are not significantly different $(\mathrm{P} \leq 0.01)$.

R: Raw; G: Germinated

Table.2 Amylose content of raw and germinated rice varieties

\begin{tabular}{|l|c|c|c|c|}
\hline \multicolumn{1}{|c|}{ Property } & \multicolumn{2}{c|}{ Amylose content (\%) } & \multicolumn{2}{c|}{ Category } \\
\cline { 2 - 5 } & \multicolumn{1}{|c|}{ Raw } & Germinated & Raw & Germinated \\
\hline Abhilash & $27.45 \pm 0.11^{\mathrm{e}}$ & $24.53 \pm 0.21^{\mathrm{d}}$ & High & Intermediate \\
\hline Ambemohar 2 & $25.27 \pm 0.10^{\mathrm{b}}$ & $22.14 \pm 0.20^{\mathrm{b}}$ & High & Intermediate \\
\hline Dodiga & $28.46 \pm 0.30^{\mathrm{g}}$ & $23.52 \pm 0.25^{\mathrm{c}}$ & High & Intermediate \\
\hline Ginasali & $26.98 \pm 0.20^{\mathrm{d}}$ & $25.44 \pm 0.30^{\mathrm{e}}$ & High & High \\
\hline Hasudi & $30.85 \pm 0.06^{\mathrm{i}}$ & $28.13 \pm 0.21^{\mathrm{h}}$ & Very High & High \\
\hline Intan & $21.77 \pm 0.21^{\mathrm{a}}$ & $19.98 \pm 0.11^{\mathrm{a}}$ & Intermediate & Low \\
\hline Jyoti & $25.94 \pm 0.15^{\mathrm{c}}$ & $22.24 \pm 0.10^{\mathrm{b}}$ & High & Intermediate \\
\hline Karikalavi & $30.42 \pm 0.10^{\mathrm{h}}$ & $26.55 \pm 0.15^{\mathrm{f}}$ & Very High & High \\
\hline Karihakkalasali & $30.59 \pm 0.15^{\mathrm{hi}}$ & $27.05 \pm 0.15^{\mathrm{g}}$ & Very High & High \\
\hline Navali & $28.03 \pm 0.11^{\mathrm{f}}$ & $25.74 \pm 0.21^{\mathrm{e}}$ & High & High \\
\hline Mean & $27.58 \pm 2.71$ & $24.53 \pm 2.46$ & & \\
\hline
\end{tabular}




\begin{tabular}{|l|c|c|c|}
\hline & SEm \pm & CD & F value \\
\hline Variety & 0.075 & 0.215 & $* *$ \\
\hline R x G & 0.033 & 0.096 & $* *$ \\
\hline Interaction & 0.106 & 0.304 & $* *$ \\
\hline
\end{tabular}

R: Raw; G: Germinated

Note: Values are mean of three replications, SEm \pm - Standard error of mean, CD - critical difference, **Significant @ 1\%. Values with same letters $(\mathrm{a}, \mathrm{b}, \mathrm{c}, \mathrm{d}, \mathrm{e}, \mathrm{f}, \mathrm{g}, \mathrm{h}, \mathrm{i})$ in the same column are not significantly different $(\mathrm{P} \leq 0.01)$.

Based on the per cent amylose content, rice varieties were classified in different categories

\begin{tabular}{|c|c|}
\hline Category & Amylose content (\%) \\
\hline Waxy & $1-2$ \\
\hline Very Low & $2-9$ \\
\hline Low & $10-20$ \\
\hline Intermediate & $20-25$ \\
\hline High & $25-30$ \\
\hline Very High & $>30$ \\
\hline
\end{tabular}

(Kumar and Khush, 1986)

Table.3 Alkali spread value of raw and germinated rice varieties

\begin{tabular}{|l|c|c|c|c|}
\hline \multirow{2}{*}{ Property } & \multicolumn{2}{c|}{ Alkali Spreading Value } & \multicolumn{2}{c|}{ Gelatinization Temperature } \\
\cline { 3 - 5 } Abariety & \multicolumn{1}{c|}{ Raw } & Germinated & Raw & Germinated \\
\hline Abhilash & $2.50 \pm 0.16^{\mathrm{bc}}$ & $3.88 \pm 0.25^{\mathrm{a}}$ & High & High Temperature \\
\hline Ambemohar 2 & $2.44 \pm 0.09^{\mathrm{bc}}$ & $4.27 \pm 0.19^{\mathrm{a}}$ & High & Intermediate \\
\hline Dodiga & $3.11 \pm 0.25^{\mathrm{d}}$ & $4.05 \pm 0.25^{\mathrm{a}}$ & High Temperature & Intermediate \\
\hline Ginasali & $2.22 \pm 0.38^{\mathrm{ab}}$ & $5.50 \pm 0.33^{\mathrm{b}}$ & High & Low \\
\hline Hasudi & $2.61 \pm 0.19^{\mathrm{c}}$ & $5.61 \pm 0.25^{\mathrm{b}}$ & High & Low \\
\hline Intan & $4.44 \pm 0.09^{\mathrm{f}}$ & $6.50 \pm 0.16^{\mathrm{c}}$ & Intermediate & Low \\
\hline Jyoti & $3.56 \pm 0.25^{\mathrm{e}}$ & $5.44 \pm 0.19^{\mathrm{b}}$ & High Temperature & Intermediate \\
\hline Karikalavi & $2.66 \pm 0.16^{\mathrm{c}}$ & $4.11 \pm 0.19^{\mathrm{a}}$ & High & Intermediate \\
\hline Karihakkalasali & $2.05 \pm 0.09^{\mathrm{a}}$ & $4.38 \pm 0.09^{\mathrm{a}}$ & High & Intermediate \\
\hline Navali & $2.44 \pm 0.09^{\mathrm{bc}}$ & $5.11 \pm 0.58^{\mathrm{b}}$ & High & Intermediate \\
\hline Mean & $2.80 \pm 0.71$ & $4.88 \pm 0.86$ & & \\
\hline
\end{tabular}

\begin{tabular}{|l|c|c|c|}
\hline & SEm \pm & CD & F value \\
\hline Variety & 0.100 & 0.285 & $* *$ \\
\hline R x G & 0.044 & 0.127 & $* *$ \\
\hline Interaction & 0.141 & 0.404 & $* *$ \\
\hline
\end{tabular}

R: Raw; G: Germinated

Note: Values are mean of three replications, SEm \pm - Standard error of mean, CD - critical difference,

**Significant @ 1\%. Values with same letters ( $\mathrm{a}, \mathrm{b}, \mathrm{c}, \mathrm{d}, \mathrm{e}, \mathrm{f})$ in the same column are not significantly different $(\mathrm{P} \leq 0.01)$. 
Based on alkali spreading values, the gelatinization temperature of rice is classified as

\begin{tabular}{|l|c|}
\hline Gelatinization temperature & Alkali spread value \\
\hline High & $1-2$ \\
\hline High Temperature & 3 \\
\hline Intermediate & $4-5$ \\
\hline Low & $6-7$ \\
\hline & (Little et al., 1958) \\
\hline
\end{tabular}

Table.4 Gel length and Gel consistency of raw and germinated rice varieties

\begin{tabular}{|l|c|c|c|c|}
\hline \multirow{2}{*}{ Property } & \multicolumn{2}{c|}{ Gel length $(\mathrm{mm})$} & \multicolumn{2}{c|}{ Gel consistency } \\
\cline { 2 - 5 } & \multicolumn{1}{|c|}{ Vawiety } & Germinated & Raw & Germinated \\
\hline Abhilash & $56.00 \pm 1.73^{\mathrm{d}}$ & $77.00 \pm 1.73^{\mathrm{de}}$ & Medium & Soft \\
\hline Ambemohar 2 & $66.00 \pm 1.73^{\mathrm{e}}$ & $74.33 \pm 2.08^{\mathrm{d}}$ & Soft & Soft \\
\hline Dodiga & $71.33 \pm 1.15^{\mathrm{f}}$ & $82.33 \pm 0.57^{\mathrm{f}}$ & Soft & Soft \\
\hline Ginasali & $54.33 \pm 3.78^{\mathrm{d}}$ & $67.00 \pm 2.64^{\mathrm{b}}$ & Medium & Soft \\
\hline Hasudi & $82.00 \pm 2.00^{\mathrm{g}}$ & $112.33 \pm 2.51^{\mathrm{h}}$ & Soft & Soft \\
\hline Intan & $88.33 \pm 3.51^{\mathrm{h}}$ & $99.33 \pm 1.15^{\mathrm{g}}$ & Soft & Soft \\
\hline Jyoti & $67.67 \pm 1.52^{\mathrm{e}}$ & $78.33 \pm 0.57^{\mathrm{e}}$ & Soft & Soft \\
\hline Karikalavi & $39.00 \pm 1.00^{\mathrm{b}}$ & $65.33 \pm 0.57^{\mathrm{b}}$ & Hard & Soft \\
\hline Karihakkalasali & $30.00 \pm 0.00^{\mathrm{a}}$ & $60.67 \pm 1.15^{\mathrm{a}}$ & Hard & Soft \\
\hline Navali & $50.67 \pm 1.15^{\mathrm{c}}$ & $70.33 \pm 0.57^{\mathrm{c}}$ & Medium & Soft \\
\hline Mean & $60.53 \pm 17.65$ & $78.70 \pm 15.49$ & & \\
\hline
\end{tabular}

\begin{tabular}{|l|c|c|c|}
\hline & SEm \pm & CD & F value \\
\hline Variety & 0.749 & 2.141 & $* *$ \\
\hline R x G & 0.335 & 0.957 & $* *$ \\
\hline Interaction & 1.059 & 3.028 & $* *$ \\
\hline
\end{tabular}

R: Raw; G:Germinated

Note: Values are mean of three replications, SEm \pm - Standard error of mean, CD - critical difference, **Significant @ 1\%. Values with same letters $(\mathrm{a}, \mathrm{b}, \mathrm{c}, \mathrm{d}, \mathrm{e}, \mathrm{f}, \mathrm{g}, \mathrm{h})$ in the same column are not significantly different $(\mathrm{P} \leq 0.01)$.

Based on gel length of rice, gel consistency is classified as

\begin{tabular}{|l|c|}
\hline Gel consistency & Gel length \\
\hline Soft & $61-100$ \\
\hline Medium & $41-60$ \\
\hline Medium Hard & $36-40$ \\
\hline Hard & $26-35$ \\
\hline
\end{tabular}



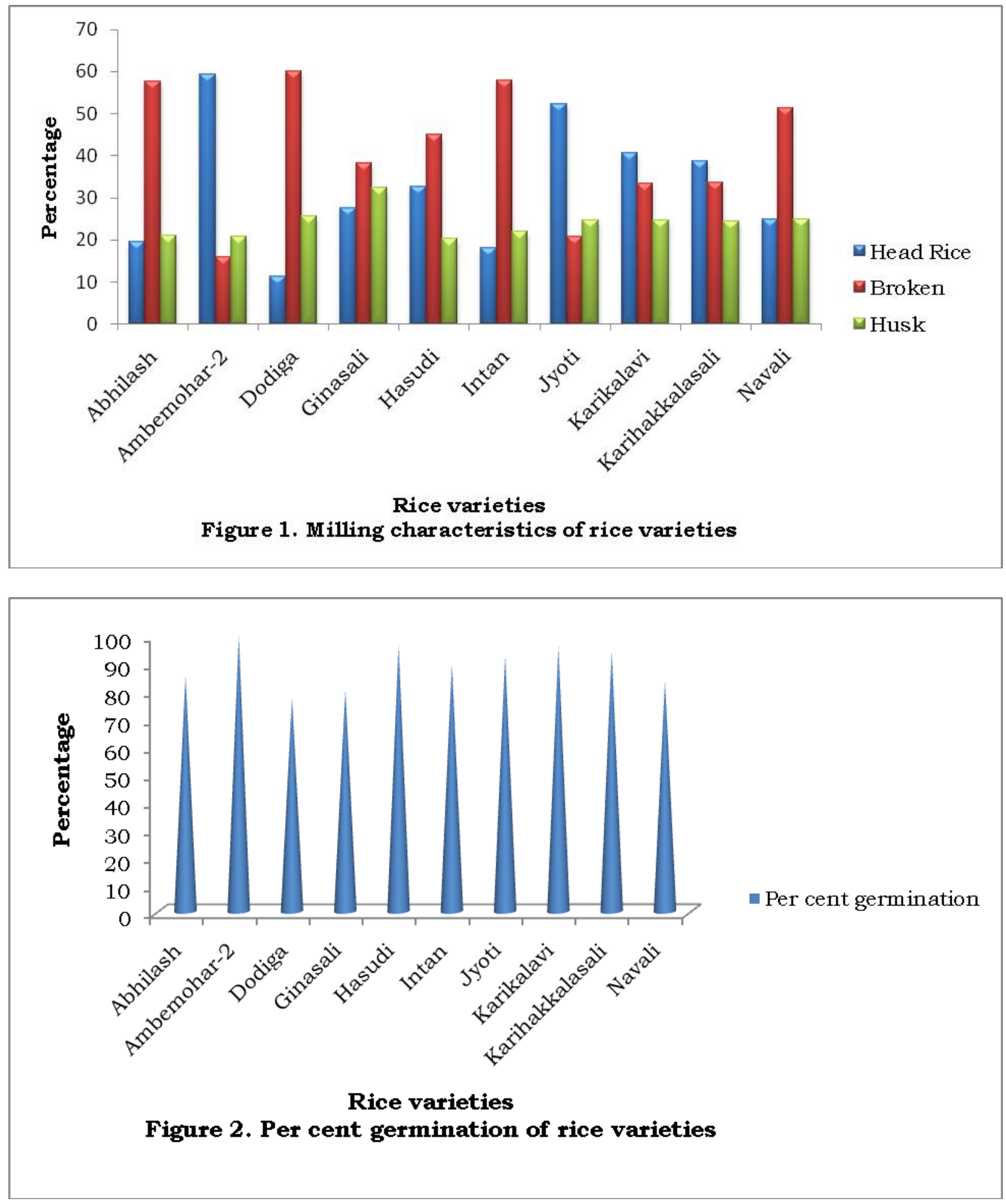

According to Quadir et al., (2012), the decrease in the bulk density may be attributed to the physical changes and leaching out of some inorganic matter and total solids during germination. Raw rice varieties had higher mean specific gravity of 1.46 compared to mean specific gravity of germinated rice varieties of 1.15 . Results showed there was significant difference in specific gravity among the rice varieties $(\mathrm{P} \leq 0.01)$. Hasudi had 
highest specific gravity of 1.93 whereas Karihakkasali had lowest specific gravity i.e., 1.15 among raw rice varieties. Similar trend was followed in case of specific gravity of germinated rice varieties. Specific gravity of rice kernel became higher or lower as the moisture content increased (1.234) or decreased (1.191) (Nagatoet.al, 1976).

The chemical properties like amylose content, alkali spread value and gel consistency of raw and germinated rice varieties are presented in Table 2, 3 and 4 respectively. The mean amylose content of raw rice i.e., 27.58 per cent was found to be higher than the mean amylose content of germinated rice varieties i.e., 24.53 per cent. There was significant difference in amylose content of different rice varieties $(\mathrm{P} \leq 0.01)$. Highest amylose content among rice varieties was recorded as 30.85 per cent in Hasudi and lowest amylose content was found in Intan (21.77 per cent). Similarly, in case of germinated rice varieties, highest amylose content of 28.13 per cent was found in Hasudi and lowest amylose content of 19.98 per cent was found in Intan. Amylose contents decreased significantly $(\mathrm{P} \leq 0.05)$ after germination ( $\mathrm{Wu}$ et al., 2013). As the results of $\alpha$-amylase activity, amylose content in germinated rice decreased. This enzyme can break down starch to shorter chains to yield dextrin and oligosaccharides (Jiamyangyuen and Ooraikul, 2008). Based on the per cent amylose content, it was found that in case of raw rice varieties, Intan (21.77 per cent) had intermediate amylose content; Abhilash (27.45 per cent), Ambemohr-2 (25.27 per cent), Dodiga (28.46 per cent), Ginasali (26.98 per cent), Jyoti (25.94 per cent), Navali (28.03 per cent) had high amylose content and Hasudi (30.85 per cent), Karikalavi (30.42 per cent), Karihakkalasali (30.59 per cent) had very high amylose content. Similarly, in case of germinated rice varieties, Intan (19.98 per cent) was found to have low amylose content; Abhilash (24.53 per cent), Ambemohr-2 (22.14 per cent), Dodiga (23.52 per cent), Jyoti (22.24 per cent) had intermediate amylose content and Ginasali (25.44 per cent), Hasudi (28.13 per cent), Karikalavi (26.55 per cent), Karihakkalasali (27.05 per cent), Navali (25.74 per cent) had high amylose content.

The mean alkali spreading value of raw rice variety was 2.80 and the mean alkali spreading value of germinated rice was 4.88 . Significant difference in alkali spreading value was found among rice varieties $(\mathrm{P} \leq 0.01)$. Rice variety, Intan had highest alkali spreading value of 4.44 whereas karihakkalasali had the lowest alkali spreading value of 2.05. Germinated rice variety, Intan had the highest alkali spreading value of 6.50 whereas germinated rice variety, Abhilash had the lowest alkali spreading value of 3.88. The alkaline spread value is used to measure gelatinization of rice and has been used for many years to categorize cooked rice properties. The results of the study conducted by Patil and Khan (2012) implied that germinated brown rice was easier to cook since it could be cooked at lower gelatinization temperature compared with that of brown rice.

Gelatinization temperature is measured using alkali spread value. Results showed that raw rice variety Intan (4.44) had intermediate gelatinization temperature; Dodiga (3.11), Jyoti (3.55) had high temperature gelatinization temperature whereas Abhilash (2.50), Ambemohr-2 (2.44), Ginasali (2.22), Hasudi (2.61), Karikalavi (2.66), Karihakkalasali (2.05), Navali (2.44) had high gelatinization temperature. In case of germinated rice varieties, Ginasali (5.50), Hasudi (5.61), Intan (6.50) were found to have low gelatinization temperature whereas Ambemohar-2 (4.27), Dodiga (4.05), Jyoti (5.44), Karikalavi (4.11), Karihakkalasali (4.38), Navali (5.11) had intermediate 
gelatinization temperature but Abhilash with alkali spread value of 3.88 was found to have high temperature gelatinization temperature. Gelatinization temperature significantly differed between raw and germinated rice varieties at 1 per cent level. Gelatinization temperature decreased on germination. The decrease in gelatinization temperatures of germinated rice might be attributed to the degradation of lipids induced by germination (Wu et.al., 2013).

The gel consistency in raw rice varieties ranged from 30.00 to $88.33 \mathrm{~mm}$ whereas gel consistency of germinated rice ranged from 60.67 to $112.33 \mathrm{~mm}$. Gel consistency among rice varieties varied significantly $(\mathrm{P} \leq 0.01)$. Based on gel length of rice, gel consistency of raw rice varietiesAmbemohr-2 $(66 \mathrm{~mm})$, Dodiga $(71.33 \mathrm{~mm})$, Hasudi $(82 \mathrm{~mm})$, Intan $(88.33 \mathrm{~mm})$, Jyoti $(67.67 \mathrm{~mm})$ were found to be soft; Abhilash $(56 \mathrm{~mm})$, Ginasali (54.33 $\mathrm{mm})$, Navali $(50.67 \mathrm{~mm})$ had medium gel consistency whereas Karikalavi (39 mm), Karihakkalasali $(30 \mathrm{~mm})$ had hard gel consistency. After germination, the gel consistency of rice ranged from 60.67 to $112.33 \mathrm{~mm}$ which showed that all germinated rice varieties had soft gel consistency. The soft and hard gel consistency values are accounted by the biallelic variability at the waxy locus (Chemutai et al., 2016). The gel consistency is expressed by the flowing distance. Gel made with germinated rice flour flowed for longer distance indicating that, upon cooking, germinated rice was softer compared to those of control (Jiamyangyuen and Ooraikul, 2008).

Thus investigation on ten rice varieties revealed that Ambemohar 2 had highest head rice yield. The per cent germination of rice was highest in Ambemohar 2 followed by Hasudi. Germination improved the physicochemical properties. Among the rice varieties, Hasudi was found to have better physico- chemical properties which could be used as a functional ingredient in different food products. Thus the results could be used in rice breeding programmes.

\section{Acknowledgement}

The authors are grateful to Department of Food Science and Nutrition, College of Rural Home Science, University of Agricultural Sciences, Dharwad, for providing the support to carry out the research.

\section{References}

Aykroyd, D.R and Doughty, J., 1964, Legumes in human nutrition, Rome. Food and Agricultural Organisation, pp. 69-80.

Bhonsle, S.J. and Sellappan, K., 2010, Grain Quality Evaluation of Traditionally Cultivated Rice Varieties of Goa, India, Recent Research in Science and Technology, 2(6): 88-97.

Chemutai, L.R., Musyoki, M.A., Kioko, W. F., Mwenda. N.S., Muriira, K. G. and Piero, N.M., 2016. Physicochemical Characterization of Selected Rice (Oryza sativa L.) Genotypes Based on Gel Consistency and Alkali Digestion. Biochem Anal Biochem., 5(3): 1-5.

Danbaba, N., Anounye, J.C., Gana, A.S., Abo, M.E. and Ukwungwu, M.N., 2011, Grain quality characteristics of Ofada rice (Oryza sativa L.): Cooking and eating quality. Int. Food Res J., 18: 629-634.

Gayin, J. Manful, J. T. and Johnson, P-N.T., 2009, Rheological and sensory properties of rice varieties from Improvement Programmes in Ghana, International Food Research Journal 16: 167-174.

Jiamyangyuen, S. and Ooraikul, B., 2008, The physico-chemical, eating and sensorial properties of germinated brown rice. 
Journal of Food, Agriculture \& Environment., 6(2): 119-124.

Kumar, I. and Khush, G.S., 1986, Gene dosage effect of amylose content in rice endosperm. Japanese J. Genet., 61: 559-568.

Little, R.R., Hilder. G.B. and Dawson, E.H, 1958, Differential effect of dilute alkali on 25 varieties of milled white rice. Cereal Chem., 35: 111-126.

Moongngarm, A. and Khomphiphatkul, E., 2011, Germination Time Dependence of Bioactive Compounds and Antioxidant Activity in Germinated Rough Rice (Oryza sativa L.).Am. J. Applied Sci., 8 (1): 15-25.

Musa, A.S.N., Umar, I.M. and Ismail, M., 2011, Physicochemical properties of germinated brown rice (Oryza sativa L.) starch. A J B., 10(33): 6281-6291.

Nagato, K., Suzuki, S. and Sado, T., 1976, Relation between the specific gravity of unhulled rice and the quality of hulled rice. Proc. Crop Sci. Soc. Japan., 45(4): 563-568.

Patil, S. B. and Khan, M. K., 2012, Some cooking properties of germinated brown rice of Indian varieties. AgricEngInt: CIGR Journal., 14(4):
156-162.

Quadir, N., Wani, S.A, Bhat, B.A, Wani, T.A. and Quraazah, A., 2012, Germination Behavior of Some Kashmiri Paddy Cultivars, J. Chem. Bio. Phy. Sci. Sec. B, 2(4): 1820-1829.

Shabbir, A.M., 2009, Biochemical And Technological Characterization of Pakistani Rice And Protein Isolates, M.Sc. (Hons.) Food Technology, National Institute Of Food Science And Technology, University Of Agriculture, Faisalabad-Pakistan: 43

Singh, R.K., Singh, U.S. and Khush, G. S., 2000, Aromatic rices. Oxford and IBH publishing Co.Pvt.Ltd. New Delhi.

Williams, P.C., Nakoul, H. and Singh, K.B. 1983, Relationship between time and some physical characteristics in chickpeas (Cicer arietinum L.).J. Sci Food Agric, 34: 492-496.

Wu, F., Chen, H., Na Yang, N., Wang, J., Duan, X., Jin, Z. and Xu, X., 2013. Effect of germination time on physicochemical properties of brownrice flour and starch from different rice cultivars. Journal of Cereal Science, 58: 263-271.

\section{How to cite this article:}

Soubhagyalaxmi P. Hiremath and Kasturiba, B. 2018. Effect of Germination on Physicochemical Properties of Rice Varieties. Int.J.Curr.Microbiol.App.Sci. 7(01): 2700-2710. doi: https://doi.org/10.20546/ijcmas.2018.701.323 